\title{
Método de Cuckow para la calibración de aerómetros: diseño y puesta a punto del equipo
}

\author{
Cáceres, J. (1), Santo, C. (1) \\ Contacto: jcaceres@latu.org.uy \\ (1) Departamento de Metrología, Laboratorio Tecnológico del Uruguay (LATU) \\ Recibido: 6/10/2008 - Aprobado: 16/12/2008
}

\begin{abstract}
Resumen
El método utilizado en el Laboratorio Tecnológico del Uruguay (LATU) para calibración de aerómetros es el "Método de Cuckow para calibración de aerómetros utilizando agua adicionada con un tensoactivo como fluido patrón". En este método se usa un tensoactivo para bajar la tensión superficial del agua destilada utilizada como fluido patrón y se verifica que la variación en la densidad de la misma, producto del agregado del tensoactivo, contribuye en forma despreciable a la incertidumbre del método y puede ser contemplada dentro de la incertidumbre aceptada para la densidad del agua destilada.

Se planificó una estrategia para bajar la incertidumbre de calibración del método; para ello se diseñó un recipiente con un sistema de termostatización con recirculación de agua por camisa, lo que aporta estabilidad térmica al sistema. Se utilizó agitación mecánica para favorecer la termostatización y evitar gradientes de densidad asociados a inhomogeneidad en la temperatura. También se realizaron mejoras en el sistema de ajuste de la lectura y formación del menisco.

Luego de estas mejoras se validó nuevamente el método y se demostró que se alcanza la meta propuesta de disminuir las incertidumbres de calibración, estando en condiciones de aplicarlo a calibración de aerómetros de precisión.

En el presente artículo se detallan las consideraciones realizadas en el diseño y puesta a punto del equipo mejorado, fundamentalmente el recipiente y el sistema de termostatización. Se plantea la construcción del recipiente a partir de tubos de vidrio disponibles en el mercado, bajando así sus costos.

Palabras clave: Cuckow, densímetros, calibración.
\end{abstract}

\begin{abstract}
Themethod usedinLaboratorio TecnológicodelUruguay(LATU)forhydrometerscalibration isthe"Cuckowmethodforhydrometers calibration using water added with a surfactant as a standard". A surfactant is used to decrease the surface tension of distilated water used as a standar and is verified that the density variation of distilated water produced by the surfactant adds a small contribution to the uncertainty of the method and can be contemplated within the accepted uncertainty for the density of distilated water. A strategy to reduce the uncertainty calibration of the method was planned; to perform it a container with a system of thermostatization with recirculating water per jacket to provide thermal stabilization to the system was designed, shaking to improve the thermostatization and avoid gradients of density associated with inhomogeneities in the temperature. Improvements were made in the adjustment system of reading and shape of the meniscus. The improved method was validated again and it was demonstrated that improvements help to reduce the uncertainties of calibration, giving the possibility to apply it for calibration of precision hydrometers. In this article considerations in designing and working conditions adjustments of the improved equipment are detailed, mainly in the container and its jacket for recirculating water of thermostatization. This raises its construction with tubes of glass available on the market and lowering the costs.
\end{abstract}

Key words: Cuckow, aerometer, calibration.

\section{Introducción}

El método utilizado en LATU para calibración de aerómetros: "Método de Cuckow para calibración de aerómetros utilizando agua adicionada con un tensoactivo como fluido patrón" fue presentado en el Simposio Metrología 2006. En aquella oportunidad se utilizó un tensoactivo para bajar la tensión superficial del agua destilada utilizada como fluido patrón. Se demostró que la variación en la densidad del agua, producto del agregado del tensoactivo, contribuye en forma despreciable a la incertidumbre del método y puede ser contemplada dentro de la incertidumbre aceptada para la densidad del agua destilada. En el trabajo anteriormente citado se presentó un cálculo de incertidumbre, demostrando que el método puede aplicarse en la calibración de la mayoría de los aerómetros disponibles en plaza. También se presentaron datos de validación del método: exactitud y precisión. Las medidas se realizaron utilizando agua y tridecano como fluidos patrón.

El equipo original utilizado (con el que se llevaron a cabo los experimentos publicados en 2006) presentaba ciertas carencias en cuanto a la homogeneidad y estabilidad en la temperatura. El recipiente de calibración consistía simplemente en una probeta sin ningún tipo de aislamiento, lo que facilitaba la transferencia de calor a través de las paredes, dificultando la obtención de menores incertidumbres. Por este motivo se planificó una estrategia para bajar la incertidumbre de calibración del método a través de la mejora del equipamiento utilizado. Para ello se diseñó un equipo de calibración con un sistema de termostatización por recirculación de agua por camisa, con agitación mecánica para favorecer la termostatizacion y evitar gradientes de densidad del liquido patrón asociados a inhomogeneidad en la temperatura. Se realizaron, además, mejoras en el sistema de ajuste de la lectura y formación del menisco. Los resultados se presentaron en IMEKO 2007. En aquella oportunidad se detallaron las mejoras introducidas al sistema original, se validó nuevamente el método mejorado y se demostró que se alcanzaba la meta propuesta de disminuir las incertidumbres de calibración, quedando en condiciones de aplicarlo a calibración de aerómetros de precisión, como alcohómetros de precisión y densímetros con divisiones $0,0001 \mathrm{~g} / \mathrm{ml}$.

El objetivo del presente artículo es detallar las consideraciones realizadas en el diseño y puesta a punto del equipo, fundamentalmente 
en el recipiente de contención del líquido patrón y su camisa de recirculación del agua de termostatización.

Las descripciones pertinentes al método de Cuckow, fundamento del método, equipos de laboratorio necesarios para su implementación, protocolo de calibración, entre otras, pueden ser encontradas en los dos artículos antes mencionados. En este artículo se describe brevemente el equipo, considerando los aerómetros disponibles y un diseño del sistema adecuado para la calibración de los mismos, que comprenden un amplio rango de dimensiones. El equipo debe cumplir, además, la meta de mantener la temperatura del líquido patrón en un rango de (20 $\pm 0,03){ }^{\circ} \mathrm{C}$.

\section{Materiales y Métodos}

\section{Descripción del equipo}

El equipo mejorado (Figura 1) consiste en un recipiente con camisa de termostatización en cuyo interior contiene el líquido de calibración donde se sumerge el aerómetro que se encuentra colgado de una balanza ubicada por encima del recipiente. Se utiliza una estructura con forma de una caja de acrílico donde puedan colocarse el recipiente en su interior y la balanza en la parte superior de la caja. Esta caja protege al equipo de corrientes de aire que puedan generarse en el laboratorio y que puedan producir tanto desestabilización térmica como oscilaciones en el aerómetro y, por lo tanto, variaciones en la lectura de la balanza.

El aerómetro es colgado de la balanza por medio de un sistema con forma de tornillo que permite el ajuste grueso para subir y bajar el instrumento hasta el punto de la escala que se quiere calibrar.

Para lograr el ajuste fino se construyó un cilindro de vidrio de 1 $\mathrm{cm}$ de diámetro y $20 \mathrm{~cm}$ de largo que se sumerge en el fluido patrón y cuyo nivel de inmersión se regula por el usuario en forma mecánica a través de un disco acoplado a un sistema de poleas y engranajes, como puede verse en la Figura 2. La variación del nivel de inmersión del cilindro permite regular el nivel del líquido en el recipiente, logrando así el ajuste final del punto de calibración; este ajuste es del orden de unos $3 \mathrm{~mm}$ aproximadamente.

\section{Diseño del recipiente}

Considerando las dimensiones de los aerómetros que comúnmente llegan al laboratorio para calibrar y en base a especificaciones (ASTM, 1995; UNIT, 2002), se determina que las dimensiones máximas esperadas para los aerómetros son:

- hv: altura del vástago del aerómetro $\rightarrow 20 \mathrm{~cm}$

- hd: longitud total del cuerpo del aerómetro (incluyendo el vástago) $\rightarrow 37 \mathrm{~cm}$

Las calibraciones se realizan por lo general en tres puntos a lo largo de la escala: al $10 \%, 50 \%$ y $90 \%$.

En los extremos, $10 \%$ y $90 \%$, se obtienen las siguientes conclusiones:

- En el punto de menor densidad calibrado, $10 \%$ escala, el aerómetro se encuentra mayormente sumergido, por lo que se debe determinar la profundidad del líquido de calibración con un margen adecuado para permitir la calibración en ese punto. Se considera que una profundidad igual a hd es suficiente para cumplir con el requerimiento.

- En el punto de mayor densidad calibrado, $90 \%$ escala, el vástago del aerómetro se encuentra mayormente afuera del líquido de calibración, por lo que debe considerarse que el largo total del recipiente debe ser tal que al restarle la altura de líquido la dimensión disponible sea mayor que hv.

Por lo expuesto, se concluye que la altura mínima total del recipiente debe ser $57 \mathrm{~cm}$, y se opta por una construcción de $65 \mathrm{~cm}$ de largo y una profundidad de líquido de $40 \mathrm{~cm}$, como una opción conservadora que atiende dimensiones de aerómetros que no se hayan tenido en cuenta.
Para determinar el diámetro del recipiente se consideraron las dimensiones estándar que ofrecen los tubos de vidrio disponibles en plaza, a partir de los cuales se construiría el recipiente. El tubo de vidrio de mayor dimensión disponible define un diámetro externo del equipo de $10 \mathrm{~cm}$ con un ancho de pared de $2 \mathrm{~mm}$. El siguiente en dimensión permite un diámetro interno de $7,6 \mathrm{~cm}$-el diámetro interno del equipo- en el cual deben disponerse el aerómetro a calibrar, el cilindro con el cual se efectuará el ajuste fino y el pt100 que se utiliza para medir la temperatura con alta exactitud.

De lo anterior se desprende que el espacio anular de la camisa tiene un ancho de $8 \mathrm{~mm}$; por dicho espacio circulará el flujo de agua de termostatización de la camisa.

En cuanto a las entradas y salidas de flujo en la camisa, se optó por una disposición en que las entradas se encuentran en la base del equipo y las salidas en la parte superior de éste. Las dos entradas (y las dos salidas) tienen direcciones opuestas entre sí, de manera que se pretende que el camino que sigue el fluido sea envolvente y ascendente, evitando que se generen caminos preferenciales con los gradientes de temperatura y los gradientes de densidad que esto ocasionaría.

Posteriormente, un estudio de homogeneidad del contenido del recipiente permite verificar si se ha logrado la meta propuesta con el diseño.

Se diseñó además una tapa de teflón para cerrar el recipiente y mantener el aire confinado dentro de éste y la porción de vástago emergente a la misma temperatura que el fluido de calibración. Esta tapa resulta de gran importancia si se desea utilizar Tridecano en lugar de agua adicionada con tensoactivo como fluido de calibración. El Tridecano es irritante de las mucosas y se debe proteger al operario de los vapores que se generen al trabajar con él. Se recomienda situar el equipo en campana para este tipo de manipulación, ya que el Tridecano puede formar mezclas explosivas con el aire.

También se verifica que para el Tridecano el coeficiente de variación de la densidad con la temperatura es mayor que el correspondiente al agua destilada, tornando más crítico el control de temperatura cuando se trabaja con este fluido como patrón.

La tapa debe contener una serie de puertos destinados a:

- Entrada en el recipiente del hilo del cual se cuelga el aerómetro de la balanza.

- Entrada en el recipiente del hilo que suspende el cilindro de ajuste fino y lo conecta con su sistema de ajuste de nivel de inmersión.

- Entrada en el recipiente del pt100 que se utiliza para medir la temperatura del fluido patrón.

Para facilitar el trabajo con los hilos se decidió la construcción de una fina ranura de unos $3 \mathrm{~mm}$ en el diámetro, cortando el borde y hasta un pequeño hoyo en el centro de ésta, donde debe centrarse el instrumento a calibrar. Se deben tomar precauciones al trabajar con Tridecano, minimizando el intercambio a través de la ranura.

\section{Determinación del régimen de funcionamiento del equipo}

Se tiene registro de que la temperatura ambiente en el laboratorio varía, a lo largo del año, en un rango comprendido entre 19 y $21^{\circ} \mathrm{C}$. Se desea mantener la temperatura en el líquido de calibración en (20 $\pm 0,03)^{\circ} \mathrm{C}$. Sobre estos datos, los de dimensionamiento del equipo y material de construcción se plantean las ecuaciones de transferencia de calor y se resuelve el sistema para funcionamiento en régimen.

Fijando la temperatura del líquido en la camisa se puede determinar el flujo de agua necesario para cumplir con el servicio, o viceversa, definiendo el flujo de agua se plantea la solución del sistema para calcular la temperatura de seteo del baño que se utiliza para recirculación de agua en la camisa.

Se prefiere fijar el flujo de agua y calcular la temperatura de seteo. Esto resulta más sencillo, ya que los caudales de agua pueden estar determinados por la bomba disponible en el laboratorio o el equipo 
de recirculación del propio baño. Además, se calcula el número de Reynolds para cada caudal de flujo disponible y se elige aquel caudal de flujo que arroje como resultado un Reynolds alto para asegurar flujo turbulento. Así se mejoran los coeficientes de transferencia de calor, y cualquier desestabilización de las condiciones ambientales se logra absorber rápidamente hasta llegar a un nuevo equilibrio. Se debe chequear que este nuevo equilibrio esté dentro de los límites especificados para el líquido de calibración.

\section{Balance de transferencia de calor en el recipiente}

$$
m \cdot C_{p} \cdot \frac{d t}{d \Theta}=0 \Rightarrow Q_{\text {liquido }}+Q_{\text {fondo }+} Q_{\text {aire }}+Q_{\text {tapateflon }}=0
$$

\section{Donde:}

- m: masa de líquido de calibración en el recipiente.

- Cp: calor específico del líquido en el recipiente.

- $t$ : temperatura del líquido en el recipiente.

- $\Theta$ : tiempo.

- Qliquido: transferencia de calor entre el líquido y la camisa por la pared lateral.

- Qfondo: transferencia de calor entre el líquido y la camisa a través del fondo del recipiente.

- Qaire: transferencia de calor entre el aire confinado y la camisa en la pared lateral.

- Qtapateflon: transferencia de calor a través de la tapa de teflón.

$$
Q_{\text {liquido }}=U l . A_{e} \cdot \Delta t
$$

Donde:

- Ul: coeficiente total de transferencia de calor referido a Ae.

- Ae: área externa lateral del recipiente en sección conteniendo líquido.

- $\Delta \mathrm{t}$ : diferencia global de temperatura entre el contenido del recipiente y el líquido en la camisa.

$$
\frac{1}{U l}=\frac{1}{h_{i}\left(\frac{A_{i}}{A_{e}}\right)}+\frac{D_{e}}{2 \cdot k} \cdot \operatorname{Ln}\left(\frac{D_{e}}{D_{0}}\right)+\frac{1}{h_{e}}
$$

Donde:

- hi: coeficiente de película referido a Ai.

- Ai: área interna lateral del recipiente en sección conteniendo líquido.

- K: conductividad térmica del vidrio.

- De: diámetro externo.

- Di: diámetro interno.

- he: coeficiente de película referido a Ae.

Análogamente se plantean las ecuaciones de transferencia de calor y se calculan los coeficientes globales de transferencia de calor para las otras tres superficies de transferencia. Las ecuaciones son similares, con las siguientes diferencias fundamentales: en Qaire debe tenerse en cuenta para el cálculo de hi que el fluido en contacto con la pared interna es el aire; en Qfondo la transferencia ocurre a través de una pared recta por lo que Uf tiene la forma:

$$
\frac{l}{U f}=\frac{l}{h_{i}}+\frac{e}{k}+\frac{l}{h_{e}}
$$

donde $\boldsymbol{e}$ es el espesor a través del cual se da la transferencia; por último en $\mathrm{Q}_{\text {tapateffon }} \mathrm{U}_{\mathrm{t}}$ tiene la forma de Uf, pero para el cálculo de hi y he el fluido en contacto es el aire y la conveccion es libre.
Fue necesario realizar algunas suposiciones, ya que no se tienen correlaciones específicas para el cálculo de los coeficientes de película involucrados en los coeficientes globales.

Para el cálculo de las propiedades de los fluidos involucrados se utilizó la temperatura promedio.

Para determinar hi de la superficie en contacto con el agua se utilizó la correlación de Chilton, Drew y Jebens (Geankoplis, 1999), desarrollada para recipientes enchaquetados utilizando el factor $\mathrm{j}$ de Sieder y Tate, y un $\mathrm{N}^{\circ}$ de Reynolds Modificado $\left(\mathrm{L}^{2} . \mathrm{N} . \rho / \mu\right)$ para agitación mecánica. Esta correlación es válida hasta $\mathrm{N}=200 \mathrm{rpm}$, donde se puede estar introduciendo aire al líquido. Para el cálculo se utilizan las dimensiones de la pastilla magnética como si fueran las de un agitador de paletas planas.

$$
\frac{h_{j \cdot} \cdot D_{j}}{k}=0.36 \cdot\left(\frac{L^{2} \cdot N \cdot \rho}{\mu}\right)^{\frac{2}{3}} \cdot\left(\frac{c \cdot \mu}{k}\right)^{\frac{1}{3}} \cdot\left(\frac{\mu}{\mu w}\right)^{0.14}
$$

Donde:

- Todas la propiedades del fluido se evalúan a la temperatura general del fluido, excepto $\mu$ w que se evalúa a temperatura de pared Tpi.

- L: longitud en pies de la paleta.

- N: número de revoluciones por segundo.

- D: densidad promedio.

- $\mu$ : viscosidad del líquido.

- Dj: diámetro interno del recipiente.

A partir de la ecuación anterior se determina hi, que se utiliza tanto para la transferencia a través de las paredes laterales como del fondo, ya que para esta última situación no existen datos disponibles.

La correlación anterior sirve para calcular el coeficiente en el recipiente, mientras que el coeficiente en la camisa puede determinarse fácilmente para sistema en régimen con la ecuación de Eagle-Ferguson (Geankoplis, 1999), usando para este caso el diámetro equivalente, dado que el flujo por la camisa puede tratarse como flujo de agua en espacio anular.

$$
h e=150(1+0.011 . t) \cdot \frac{v^{0.8}}{D^{0.2}}
$$

Donde:

- D: diámetro equivalente en pulgadas.

- v: velocidad de flujo en pie/s.

- he: coeficiente pelicular en Btu/(h.pie $\left.{ }^{2}{ }^{\circ} \mathrm{F}\right)$.

- $\mathrm{t}$ : temperatura de la corriente $/{ }^{\circ} \mathrm{F}$.

De esta manera se determina he. Para la determinación del coeficiente de película por convección libre del aire que se encuentra confinado encima del liquido dentro del recipiente de calibración se utiliza una ecuación semiempírica (Geankoplis, 1999) que determina los coeficientes de película para placas planas o cilindros verticales.

A partir de los balances anteriores se determina la temperatura en la camisa necesaria para mantener la temperatura en el recipiente en el valor deseado. Para determinar la temperatura de seteo del baño necesaria para mantener la temperatura en la camisa en el valor deseado debe plantearse un balance análogo al detallado, pero para el fluido que circula por la camisa.

\section{Resultados}

Para predecir el funcionamiento del equipo se elabora una planilla de cálculo (Figura 3), en la que se ingresan los datos de condiciones ambientales ( $\mathrm{P}, \mathrm{HR}$ y $\mathrm{T})$ y los parámetros de funcionamiento del 
equipo (agitación (rpm), largo pastilla y flujo de agua por camisa). La planilla arroja como resultado la temperatura del agua en la camisa necesaria para mantener el líquido de calibración a $20^{\circ} \mathrm{C}$. En la Tabla 1 se muestran las condiciones de operación para un conjunto dado de condiciones ambientales determinadas.

Se construye el equipo que se muestra en la Figura 4 y se verifica que las condiciones de operación cumplan con lo esperado.

Se observa que la estabilidad es la deseada, realizándose un estudio de homogeneidad y un estudio de la estabilidad en el tiempo que se resume en la Tabla 2. calibración estables en un período de tiempo suficiente para llevar a cabo la calibración de densímetros, minimizando así los aportes a la incertidumbre debidos a la estabilidad y homogeneidad del baño. Se observa que las transferencias de calor son bajas a causa de los bajos gradientes térmicos y pequeñas áreas de transferencia, garantizando la estabilidad deseada para poder reducir la incertidumbre del método. Esto era esperable de acuerdo a los balances planteados. Se demuestra que el diseño de equipo es adecuado, ya que se logra disminuir la incertidumbre expandida del método de $0,000088 \mathrm{~g} / \mathrm{ml} \mathrm{a} 0,000028 \mathrm{~g} /$ $\mathrm{ml}$, tal como se expresa en el artículo publicado en IMEKO 2007, haciendo posible la calibración de aerómetros de precisión.

\begin{tabular}{|c|c|c|c|c|}
\hline $\begin{array}{c}\text { Condiciones } \\
\text { ambientales }\end{array}$ & Condiciones de operación & $\begin{array}{c}\text { Condiciones dentro del } \\
\text { recipiente }\end{array}$ & hi=551,529 J/(s.m2. $\left.{ }^{\circ} \mathrm{C}\right)$ & $\begin{array}{c}\text { Cálculo de las condiciones } \\
\text { de operación }\end{array}$ \\
\hline $\mathrm{P}=101325 \mathrm{hPa}$ & $\mathrm{N}=150 \mathrm{rpm}$ & $\mathrm{P}=101325 \mathrm{hPa}$ & $\begin{array}{c}\mathrm{he}=18,511 \mathrm{~J} /(\mathrm{s} . \mathrm{m} 2 . \\
\left.{ }^{\circ} \mathrm{C}\right)\end{array}$ & $\Sigma \mathrm{Q}=0,00847 \mathrm{~J} / \mathrm{s}$ \\
\hline $\mathrm{H}=50 \% \mathrm{HR}$ & $\mathrm{Q}=7$ litros/minutos & $\mathrm{H}=80 \% \mathrm{HR}$. & hai $=0,21 \mathrm{~J} /\left(\mathrm{s} . \mathrm{m} 2 .{ }^{\circ} \mathrm{C}\right)$ & $\mathrm{T}_{\text {liq calibracion }}=20{ }^{\circ} \mathrm{C}$ \\
\hline $\mathrm{T}=19{ }^{\circ} \mathrm{C}$ & $\begin{array}{c}\text { Largo pastilla }=2,2 \\
\mathrm{~mm} .\end{array}$ & $\mathrm{T}=20{ }^{\circ} \mathrm{C}$. & $\begin{array}{c}\text { hae }=1,12 \mathrm{~J} /(\mathrm{s} \cdot \mathrm{m} 2 . \\
\left.{ }^{\circ} \mathrm{C}\right)\end{array}$ & $\mathrm{T}_{\text {camisa }}=20,005^{\circ} \mathrm{C}$ \\
\hline
\end{tabular}

Tabla 1. Resultados calculados.

\section{Referencias}

\begin{tabular}{|c|c|c|c|c|c|}
\hline 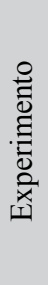 & 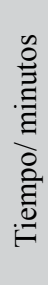 & 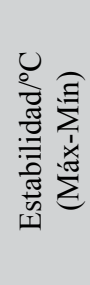 & 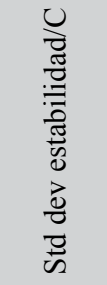 & 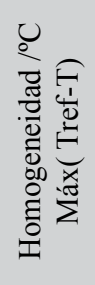 & 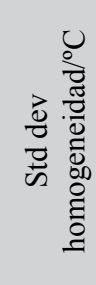 \\
\hline 1 & 6 & 0,004 & 0,0016 & 0,008 & 0,0013 \\
\hline 2 & 30 & 0,009 & 0,005 & 0,008 & 0,0038 \\
\hline 3 & 70 & 0,012 & 0,005 & 0,008 & 0,0068 \\
\hline
\end{tabular}

Tabla 2. Resultados estudio homogeneidad.

\section{Conclusiones}

Se comprueba que el equipo funciona de acuerdo a lo esperado y propuesto por el diseño. Los estudios de homogeneidad y estabilidad permiten observar que el sistema ofrece condiciones de
- ASTM INTERNATIONAL (United States). E100: Standard specification for ASTM hydrometers. West Conshohocken: ASTM, 1995.

- GEANKOPLIS, Christie J. Procesos de transporte y operaciones unitarias. 3a ed. México: Continental, 1999.

- INSTITUTO URUGUAYO DE NORMAS TÉCNICAS (Uruguay). UNIT-ISO 649-2: Density hidrometers for general purposes. Montevideo: UNIT, 2002.

- SANTO, Claudia; CACERES, Joselaine. Improvement of Cuckow method for calibration of aerometers in LATU. Comparison of the performance of three different liquids as hydrostatic weighing standards. En: IMEKO. International Measurement Confederation. IMEKO, (Yucatán 26-28 de noviembre de 2007). Yucatán: IMEKO, 2007.

- SANTO, Claudia; CACERES, Joselaine. Método de Cuckow para la calibración de aerómetros utilizando agua destilada adicionada con un tensoactivo como fluído patrón. En: CENAM. Simposio Metrología. Mediciones confiables para el desarrollo de México, (Santiago de Querétaro 25-27 de octubre de 2006). Santiago de Querétaro: CENAM, 2006. 


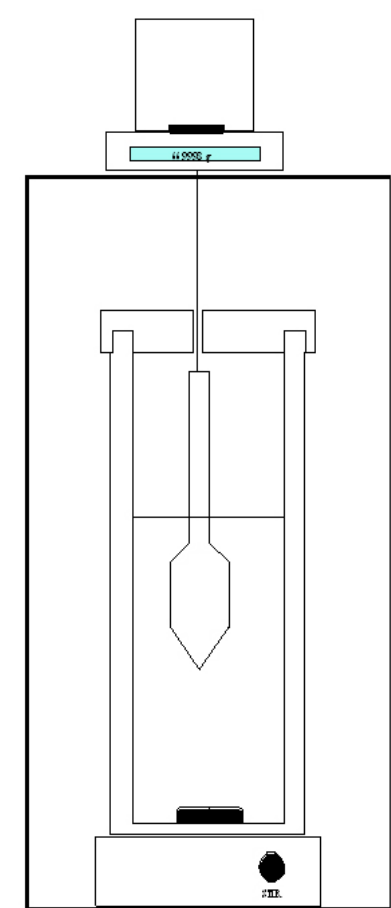

Figura 1. Esquema del equipo de trabajo. Se observa la estructura que ofrece soporte a la balanza y resguardo al recipiente con camisa de termostatización.

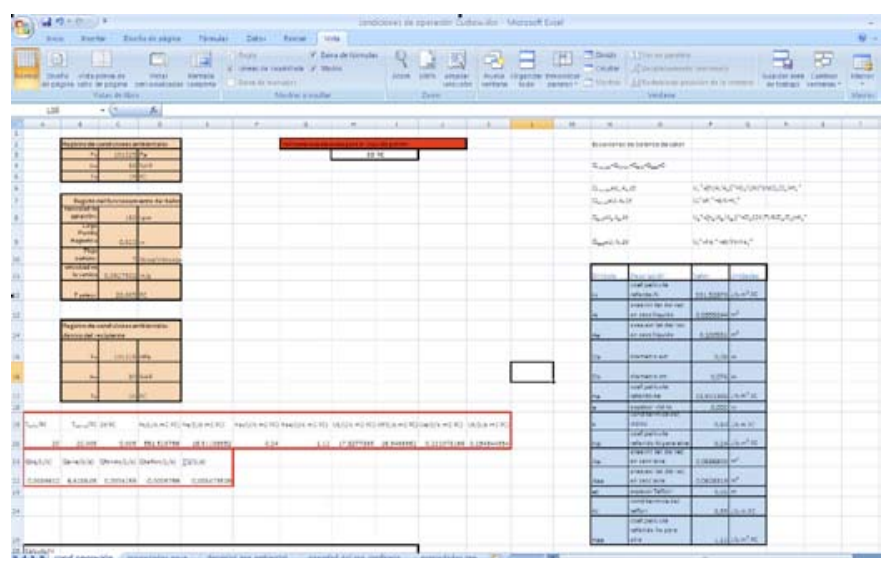

Figura 3. Planilla de cálculo que se utiliza para estimar los coeficientes de transferencia de calor y una rápida estimación de las condiciones de operación cuando varían las condiciones ambientales

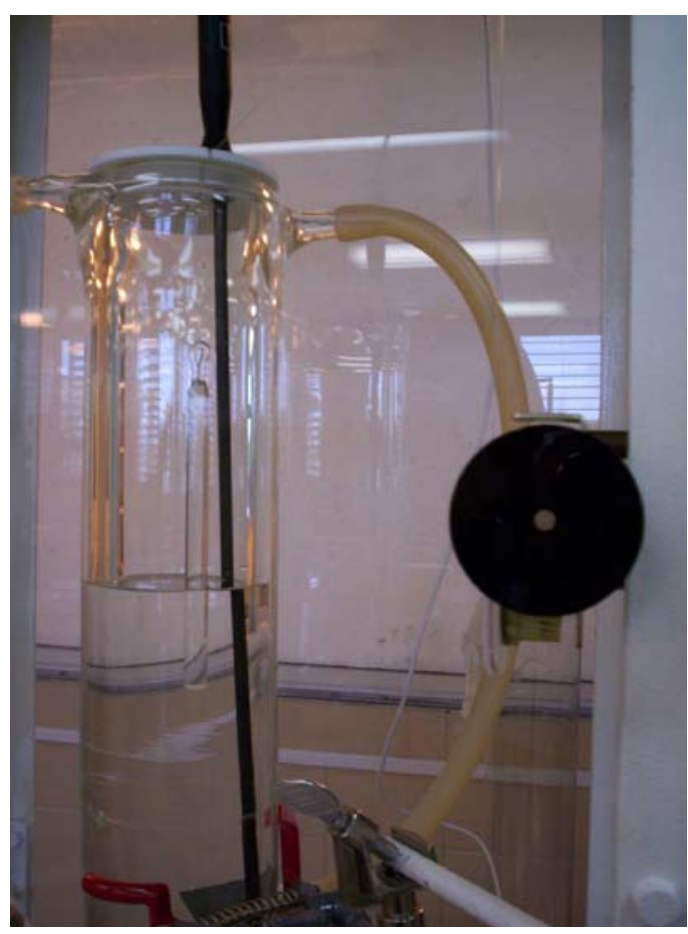

Figura 2. Detalle del cilindro de ajuste fino y poleas de ajuste de nivel.

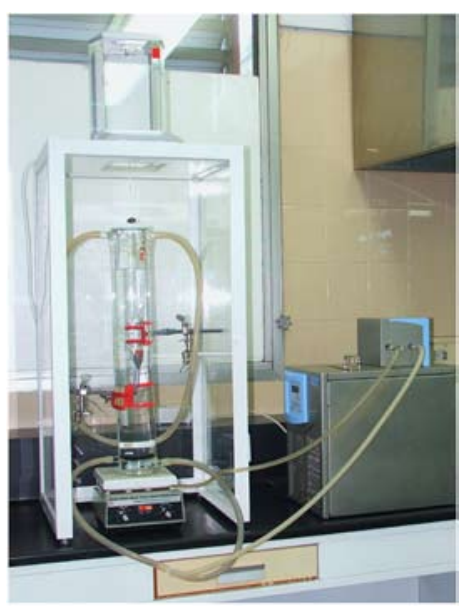

Figura 4. Imagen del equipo construido y armado para la calibración de densímetros. 